\title{
gr-n-ideals in graded commutative rings
}

\author{
Khaldoun Al-Zoubi \\ Department of Mathematics and \\ Statistics, Jordan University of Science \\ and Technology, Irbid, Jordan \\ email: kfzoubi@just.edu.jo
}

\author{
Farah Al-Turman \\ Department of Mathematics, \\ University of Jordan, Amman, Jordan \\ email: farahgha13@gmail.com
}

\author{
Ece Yetkin Celikel \\ Department of Mathematics, \\ Faculty of Art and Science, \\ Gaziantep University Gaziantep, Turkey \\ email: yetkinece@gmail.com
}

\begin{abstract}
Let $\mathrm{G}$ be a group with identity $e$ and let $\mathrm{R}$ be a $\mathrm{G}$-graded ring. In this paper, we introduce and study the concept of gr-n-ideals of $R$. We obtain many results concerning gr- $n$-ideals. Some characterizations of $\mathrm{gr}$ - $\mathrm{n}$-ideals and their homogeneous components are given.
\end{abstract}

\section{Introduction and preliminaries}

Throughout this article, rings are assumed to be commutative with $1 \neq 0$. Let $\mathrm{R}$ be a ring, I be a proper ideal of $\mathrm{R}$. By $\sqrt{\mathrm{I}}$, we mean the radical of I which is $\left\{r \in R: r^{n} \in I\right.$ for some positive integer $\left.n\right\}$. In particular, $\sqrt{0}$ is the set of nilpotent elements in R. Recall from [11] that a proper ideal $I$ of $R$ is said to be an $n$-ideal if whenever $a, b \in R$ and $a b \in I$ with $a \notin \sqrt{0}$ implies $b \in I$. For $a \in R$, we define $\operatorname{Ann}(a)=\{r \in R: r a=0\}$.

The scope of this paper is devoted to the theory of graded commutative rings. One use of rings with gradings is in describing certain topics in algebraic 
geometry. Here, in particular, we are dealing with gr-n-ideals in a G-graded commutative ring.

First, we recall some basic properties of graded rings which will be used in the sequel. We refer to [6]-[8] for these basic properties and more information on graded rings.

Let $\mathrm{G}$ be a group with identity e. A ring $\mathrm{R}$ is called graded (or more precisely, G-graded ) if there exists a family of subgroups $\left\{R_{g}\right\}$ of $R$ such that $R=\oplus_{g \in G} R_{g}$ (as abelian groups) indexed by the elements $g \in G$, and $R_{g} R_{h} \subseteq R_{g h}$ for all $g, h \in G$. The summands $R_{g}$ are called homogeneous components and elements of these summands are called homogeneous elements. If $a \in R$, then $a$ can be written uniquely $a=\sum_{g \in G} a_{g}$ where $a_{g}$ is the component of $a$ in $R_{g}$. Also, we write $h(R)=\cup_{g \in G} R_{g}$. Let $R=\underset{g \in G}{\oplus} R_{g}$ be a G-graded ring. An ideal I of $R$ is said to be a graded ideal if $I=\oplus_{g \in G}\left(I \cap R_{g}\right):=\oplus_{g \in G} I_{g}$. An ideal of a graded ring need not be graded.

If $I$ is a graded ideal of $R$, then the quotient ring $R / I$ is a G-graded ring. Indeed, $R / I=\bigoplus_{g \in G}(R / I)_{g}$ where $(R / I)_{g}=\left\{x+I: x \in R_{g}\right\}$. A G-graded ring $R$ is called a graded integral domain (gr-integral domain) if whenever $r_{g}, s_{h} \in h(R)$ with $r_{g} s_{h}=0$, then either $r_{g}=0$ or $s_{h}=0$.

The graded radical of a graded ideal I, denoted by $\mathrm{Gr}(\mathrm{I})$, is the set of all $x=\sum_{g \in G} x_{g} \in R$ such that for each $g \in G$ there exists $n_{g} \in \mathbb{N}$ with $x_{g}^{n_{g}} \in I$. Note that, if $r$ is a homogeneous element, then $r \in \operatorname{Gr}(\mathrm{I})$ if and only if $r^{\mathrm{n}} \in \mathrm{I}$ for some $\mathrm{n} \in \mathbb{N}$, (see [10].)

Let $\mathrm{R}$ be a G-graded ring. A graded ideal $\mathrm{I}$ of $\mathrm{R}$ is said to be a graded prime (gr-prime) if $\mathrm{I} \neq \mathrm{R}$; and whenever $\mathrm{r}_{g}, s_{\mathrm{h}} \in \mathrm{h}(\mathrm{R})$ with $\mathrm{r}_{\mathrm{g}} \mathrm{s}_{\mathrm{h}} \in \mathrm{I}$, then either $r_{g} \in I$ or $s_{h} \in I$, (see [10].)

The concepts of graded primary ideals and graded weakly primary ideals of a graded ring have been introduced in [9] and [5], respectively. Let I be a proper graded ideal of a graded ring $\mathrm{R}$. Then $\mathrm{I}$ is called a graded primary (gr-primary) (resp. graded weakly primary) ideal if whenever $r_{g}, s_{h} \in h(R)$ and $r_{g} s_{h} \in I$ (resp. $0 \neq r_{g} s_{h} \in I$ ), then either $r_{g} \in I$ or $s_{h} \in \operatorname{Gr}(I)$.

Graded 2-absorbing and graded weakly 2-absorbing ideals of a commutative graded rings have been introduced in [2]. According to that paper, I is said to be a graded 2-absorbing (resp. graded weakly 2-absorbing) ideal of $\mathrm{R}$ if whenever $r_{g}, s_{h}, t_{i} \in h(R)$ with $r_{g} s_{h} t_{i} \in I$ (resp. $\left.0 \neq r_{g} s_{h} t_{i} \in I\right)$, then $r_{g} s_{h} \in I$ or $r_{g} t_{i} \in I$ or $s_{h} t_{i} \in I$.

Then the graded 2-absorbing primary and graded weakly 2-absorbing primary ideals defined and studied in [4]. A graded ideal I is said to be a graded 2-absorbing primary (resp. graded weakly 2-absorbing primary) ideal of $\mathrm{R}$ if 
whenever $r_{g}, s_{h}, t_{i} \in h(R)$ with $r_{g} s_{h} t_{i} \in I$ (resp. $\left.0 \neq r_{g} s_{h} t_{i} \in I\right)$, then $r_{g} s_{h} \in I$ or $r_{g} t_{i} \in \mathrm{Gr}(\mathrm{I})$ or $s_{h} t_{i} \in \mathrm{Gr}(\mathrm{I})$.

Recently, R. Abu-Dawwas and M. Bataineh in [1] introduced and studied the concepts of graded $r$-ideals of a commutative graded rings. A proper graded ideal $I$ of $R$ is said to be a graded $r$-ideal ( $g r-r$-ideal) of $R$ if whenever $r_{g}, s_{h} \in$ $h(R)$ such that $r_{g} s_{h} \in I$ and $\operatorname{Ann}(a)=\{0\}$, then $s_{h} \in I$.

In this paper, we introduce the concept of graded $n$-ideals ( $g r-n$-ideals) and investigate the basic properties and facts concerning gr-n-ideals.

\section{Results}

Definition 1 Let $\mathrm{R}$ be a $\mathrm{G}$-graded ring. A proper graded ideal $\mathrm{I}$ of $\mathrm{R}$ is called a graded $n$-ideal of $R$ if whenever $r_{g}, s_{h} \in h(R)$ with $r_{g} s_{h} \in I$ and $r_{g} \notin \operatorname{Gr}(0)$, then $\mathrm{r}_{\mathrm{g}} \in \mathrm{I}$. In short, we call it a gr-n-ideal.

Example 1 (i) Suppose that $(\mathrm{R}, \mathrm{M})$ is a graded local ring with unique graded prime ideal. Then every graded ideal is a gr-n-ideal.

(ii) In any graded integral domain $\mathrm{D}$, the graded zero ideal is a gr-n-ideal.

(iii) Any graded ring $\mathrm{R}$ need not have a gr-n-ideal. For instance, let $\mathrm{G}=\mathbb{Z}_{2}$, $\mathrm{R}=\mathbb{Z}_{6}$ be a $\mathrm{G}$-graded ring with $\mathrm{R}_{0}=\mathbb{Z}_{6}$ and $\mathrm{R}_{1}=\{0\}$. Then $\mathrm{R}$ has not any gr-n-ideal.

Lemma 1 Let $\mathrm{R}$ be a $\mathrm{G}$-graded ring and $\mathrm{I}$ be a graded ideal of $\mathrm{R}$. If $\mathrm{I}$ is a gr-n-ideal of $\mathrm{R}$, then $\mathrm{I} \subseteq \mathrm{Gr}(0)$.

Proof. Assume that I is a gr-n-ideal and $I \nsubseteq \operatorname{Gr}(0)$. Then there exists $r_{g} \in$ $h(R) \cap I$ such that $r_{g} \notin \operatorname{Gr}(0)$. Since $r_{g} 1=r_{g} \in I$ and I is a gr-n-ideal, we get $1 \in \mathrm{I}$, so $\mathrm{I}=\mathrm{R}$, a contradiction. Hence $\mathrm{I} \subseteq \mathrm{Gr}(0)$.

Theorem 1 Let $\mathrm{R}$ be a G-graded ring and $\mathrm{I}$ be a gr-prime ideal of $\mathrm{R}$. Then $\mathrm{I}$ is a gr-n-ideal of $\mathrm{R}$ if and only if $\mathrm{I}=\mathrm{Gr}(0)$.

Proof. Assume that I is a gr-prime ideal of R. It is easy to see $\operatorname{Gr}(0) \subseteq$ $\operatorname{Gr}(\mathrm{I})=\mathrm{I}$. If I is a $\mathrm{gr}$-n-ideal of $\mathrm{R}$, by Lemma 1 , we have $\mathrm{I} \subseteq \operatorname{Gr}(0)$ and so $I=\operatorname{Gr}(0)$. For the converse, assume that $I=G r(0)$. Let $r_{g}, s_{h} \in h(R)$ such that $r_{g} s_{h} \in I$ and $r_{g} \notin \operatorname{Gr}(0)$. Since I is a gr-prime ideal and $r_{g} \notin \operatorname{Gr}(0)=I$, we get $s_{h} \in I$. 
Corollary 1 Let $\mathrm{R}$ be a $\mathrm{G}$-graded ring. Then $\mathrm{Gr}(0)$ is a gr-n-ideal of $\mathrm{R}$ if and only if it is a $\mathrm{gr}$-prime ideal of $\mathrm{R}$.

Proof. Assume that $\operatorname{Gr}(0)$ is a gr-n-ideal of R. Let $r_{g}, s_{h} \in h(R)$ such that $r_{g} s_{h} \in \operatorname{Gr}(0)$ and $r_{g} \notin \operatorname{Gr}(0)$. Then $s_{h} \in \operatorname{Gr}(0)$ as $\operatorname{Gr}(0)$ is a gr-n-ideal of R. Hence $\operatorname{Gr}(0)$ is a $g r$-prime ideal of R. Conversely, Assume that $\operatorname{Gr}(0)$ is a gr-prime ideal of $R$, by Theorem 1, we conclude that $\operatorname{Gr}(0)$ is a $g r$-n-ideal of R.

The following theorem give us a characterization of gr-n-ideal of a graded rings.

Theorem 2 Let $\mathrm{R}$ be a graded ring and $\mathrm{I}$ be a proper graded ideal of $\mathrm{R}$. Then the following statements are equivalent:

(i) I is a gr-n-ideal of R.

(ii) $\mathrm{I}=\left(\mathrm{I}:_{\mathrm{R}} \mathrm{r}_{\mathrm{g}}\right)$ for every $\mathrm{r}_{\mathrm{g}} \in \mathrm{h}(\mathrm{R})-\mathrm{Gr}(0)$.

(iii) For every graded ideals $\mathrm{J}$ and $\mathrm{K}$ of $\mathrm{R}$ such that $\mathrm{JK} \subseteq \mathrm{I}$ and $\mathrm{J} \cap(\mathrm{h}(\mathrm{R})-$ $\mathrm{Gr}(0)) \neq \emptyset$ implies $\mathrm{K} \subseteq \mathrm{I}$.

Proof. (i) $\Rightarrow$ (ii) Assume that I is a gr-n-ideal of R. Let $r_{g} \in h(R)-G r(0)$. Clearly, $I \subseteq\left(I: R r_{g}\right)$. Now, Let $s=\sum_{h \in G} s_{h} \in\left(I: R r_{g}\right)$. This yields that $r_{g} s_{h} \in I$ for each $h \in G$. Since I is a gr-n-ideal of $R$ and $r_{g} \in h(R)-G r(0)$, we have $s_{h} \in I$ for each $h \in G$ and so $s \in I$. This implies that $\left(I: R r_{g}\right) \subseteq I$. Therefore, $\mathrm{I}=\left(\mathrm{I}:_{\mathrm{R}} \mathrm{r}_{\mathrm{g}}\right)$.

(ii) $\Rightarrow$ (iii) Assume that $J K \subseteq$ I with $J \cap(h(R)-G r(0)) \neq \emptyset$ for graded ideals $J$ and $K$ of $R$. Then there exists $r_{g} \in J \cap h(R)$ such that $r_{g} \notin G r(0)$. Hence $r_{g} K \subseteq I$, it follows that $K \subseteq\left(I: R r_{g}\right)$. By our assumption, we obtain $\mathrm{K} \subseteq\left(\mathrm{I}: \mathrm{R} \mathrm{r}_{\mathrm{g}}\right)=\mathrm{I}$.

(iii) $\Rightarrow$ (i) Let $r_{g}, s_{h} \in h(R)$ such that $r_{g} s_{h} \in I$ and $r_{g} \notin G r(0)$. Let $J=r_{g} R$ and $K=s_{h} R$ be two graded ideals of $R$ generated by $r_{g}$ and $s_{h}$, respectively. Then JK $\subseteq \mathrm{I}$. By our assumption, we obtain, $\mathrm{K} \subseteq \mathrm{I}$ and so $s_{\mathrm{h}} \in \mathrm{I}$. Thus $\mathrm{I}$ is a gr-n-ideal of R.

Theorem 3 Let $\mathrm{R}$ be a $\mathrm{G}$-graded ring and $\left\{\mathrm{I}_{\alpha}\right\}_{\alpha \in \Lambda}$ be a non empty set of gr-n-ideals of $R$. Then $\cap_{i \in \Delta} I_{i}$ is gr-n-ideal of $R$.

Proof. Clearly, $\cap_{\alpha \in \Lambda} I_{\alpha}$ is a graded ideal of R. Let $r_{g}, s_{h} \in h(R)$ such that $r_{g} s_{h} \in \cap_{\alpha \in \Lambda} I_{\alpha}$ and $r_{g} \notin \operatorname{Gr}(0)$. Then $r_{g} s_{h} \in I_{\alpha}$ for every $\alpha \in \Lambda$. Since $I_{\alpha}$ is a gr-n-ideal of $R$, we have $s_{h} \in I_{\alpha}$ for every $\alpha \in \Lambda$ thus $s_{h} \in \cap_{\alpha \in \Lambda} I_{\alpha}$. 
Theorem 4 Let $\mathrm{R}$ be a $\mathrm{G}$-graded ring and $\mathrm{I}$ be a graded ideal of $\mathrm{R}$. If $\mathrm{I}$ is a gr-n-ideal of $\mathrm{R}$, then $\mathrm{I}$ is a gr-r-ideal of $\mathrm{R}$.

Proof. Assume that I is a gr-n-ideal of R. Let $r_{g}, s_{h} \in h(R)$ such that $r_{g} s_{h} \in I$ and $\operatorname{ann}\left(r_{g}\right)=0$. Since $\operatorname{ann}\left(r_{g}\right)=0, r_{g} \notin \operatorname{Gr}(0)$. Then $s_{h} \in I$ as I is a gr-n-ideal. Thus I is a $g r-r$-ideal of $R$.

Remark 1 It is easy to see that every graded nilpotent element is also a graded zero divisor. So graded zero divisors and graded nilpotent elements are equal in case $\langle 0\rangle$ is a graded primary ideal of $\mathrm{R}$. Thus the gr-n-ideals and $\mathrm{gr}-\mathrm{r}-$ ideals are equivalent in any graded commutative ring whose graded zero ideal is graded primary.

Recall that a G-graded ring $R$ is called a G-graded reduced ring if $r^{2}=0$ implies $r=0$ for any $r \in h(R)$; i.e. $\operatorname{Gr}(0)=0$.

Theorem 5 Let $\mathrm{R}$ be a G-graded ring. Then the following hold:

(i) Any G-graded reduced ring $\mathrm{R}$, which is not graded integral domain, has no gr-n-ideal.

(ii) If $\mathrm{R}$ is a $\mathrm{G}$-graded reduced ring, then $\mathrm{R}$ is a graded integral domain if and only if 0 is a gr-n-ideal.

Proof. ( $i$ ) Let $R$ be a G-graded reduced ring such that $R$ is not graded integral domain. Assume that there exists a gr-n-ideal I of R. Since $R$ is a G-graded reduced ring, $\operatorname{Gr}(0)=0$. By Lemma 1, we get, $\mathrm{I} \subseteq \operatorname{Gr}(0)=0$ and $\operatorname{so} \operatorname{Gr}(0)=$ $0=\mathrm{I}$. Since $\operatorname{Gr}(0)=0$ is not gr-prime ideal of $\mathrm{R}$, by Corollary 1, we get $\mathrm{I}=\mathrm{Gr}(0)$ is not a $\mathrm{gr}$-n-ideal, a contradiction.

(ii) Assume that $R$ is a $G$-graded reduced ring. If $R$ is a graded integral domain, then $\operatorname{Gr}(0)=0$ is a gr-prime ideal, and hence by Corollary 1, $0=$ $\operatorname{Gr}(0)$ is a $\mathrm{gr}$-n-ideal of $\mathrm{R}$. For the converse if 0 is a $\mathrm{gr}$ - $n$-ideal of $R$, then by part $(i) R$ is a graded integral domain.

Theorem 6 Let $\mathrm{R}$ be a $\mathrm{G}$-graded ring, I be a gr-n-ideal of $\mathrm{R}$ and $\mathrm{t}_{\mathrm{g}} \in \mathrm{h}(\mathrm{R})-\mathrm{I}$. Then $\left(\mathrm{I}: \mathrm{R} \mathrm{t}_{\mathrm{g}}\right)$ is a gr-n-ideal of $\mathrm{R}$.

Proof. By [9, Proposition 1.13], ( $I:_{R} t_{g}$ ) is a graded ideal. Since $t_{g} \notin I$, $\left(I: R t_{g}\right) \neq R$. Now, let $r_{h}, s_{\lambda} \in h(R)$ such that $r_{h} s_{\lambda} \in\left(I:_{R} t_{g}\right)$ and $r_{h} \notin$ $\operatorname{Gr}\left(\left(I: R t_{g}\right)\right)$. Then $r_{h} s_{\lambda} t_{g} \in I$. Since I is a gr-n-ideal of $R$ and $r_{h} \notin \operatorname{Gr}(0)$, we get $s_{\lambda} t_{g} \in I$. This yields that $s_{\lambda} \in\left(I: R t_{g}\right)$. Therefore, ( $\left.I: R t_{g}\right)$ is a gr-n-ideal of $R$. 
Theorem 7 Let $\mathrm{R}$ be G-graded ring and $\mathrm{I}$ be a graded ideal of $\mathrm{R}$. If $\mathrm{I}$ is a maximal gr-n-ideal of $\mathrm{R}$, then $\mathrm{I}=\mathrm{Gr}(0)$.

Proof. Assume that I is a maximal gr-n-ideal of R. Let $r_{g}, s_{h} \in h(R)$ such that $r_{g} s_{h} \in I$ and $r_{g} \notin I$. Since I is a gr-n-ideal and $r_{g} \notin I$, by Theorem 6 , we have $\left(I:_{R} r_{g}\right)$ is a $g r-n$-ideal. Thus $s_{h} \in\left(I:_{R} r_{g}\right)=I$ by maximality of $I$. This yields that $I$ is a $g r$-prime ideal of $R$. By Theorem 1 , we get $I=\operatorname{Gr}(0)$.

Lemma 2 Let $\mathrm{R}$ be a $\mathrm{G}$-graded ring and $\left\{\mathrm{I}_{\mathrm{i}}: \mathrm{i} \in \Lambda\right.$ \} be a directed collection of gr-n-ideals of $\mathrm{R}$. Then $\mathrm{I}=\cup_{\mathrm{i} \in \Lambda} \mathrm{I}_{\mathrm{i}}$ is a gr-n-ideal of $\mathrm{R}$.

Proof. Suppose that $r_{g} s_{h} \in I$ and $r_{g} \notin G r(0)$ for some $r_{g}, s_{h} \in h(R)$. Hence $r_{g} s_{h} \in I_{k}$ for some $k \in \Lambda$. Since $I_{k}$ is a gr- $n$-ideal of $R$, we conclude that $s_{h} \in I_{k} \subseteq \cup_{i \in \Lambda} I_{i}=I$. Thus I is a gr-n-ideal.

Theorem 8 Let $\mathrm{R}$ be a G-graded ring. Then the following statements are equivalent:

(i) $\operatorname{Gr}(0)$ is a gr-prime ideal of $\mathrm{R}$.

(ii) There exists a gr-n-ideal of $\mathrm{R}$.

Proof. (i) $\Rightarrow$ (ii) It is clear by Corollary 1 .

(ii) $\Rightarrow$ (i) First we show that $R$ has a maximal gr-n-ideal. Let $D$ be the set of all gr-n-ideals of $R$. Then by our assumption, $D \neq \emptyset$. Since $D$ is a poset by the set inclusion, take a chain $\mathrm{I}_{1} \subseteq \mathrm{I}_{2} \subseteq \cdots$ in $\mathrm{D}$. We conclude that the upper bound of this chain is $I=\cup_{i=1}^{\infty} I_{i}$ by Lemma 2. Then $D$ has a maximal element which is a maximal gr-n-ideal. Thus that ideal is $\operatorname{Gr}(0)$ by Corollary 1 and Theorem 7 .

In view of Lemma 1 and Theorem 8, we have the following result.

Theorem 9 Let $\mathrm{R}$ be a G-graded ring and $\mathrm{I}$ a graded ideal of $\mathrm{R}$ such that $\mathrm{I} \subseteq \mathrm{Gr}(0)$.

(i) I is a gr-n-ideal if and only if I is a gr-primary ideal.

(ii) If I is a gr-n-ideal, then I is a graded weakly primary (so graded weakly 2-absorbing primary) and graded 2-absorbing primary ideal.

(iii) If $\mathrm{Gr}(0)$ is gr-prime, then I is a graded weakly 2-absorbing primary ideal if and only if $\mathrm{I}$ is a graded 2-absorbing primary ideal of $\mathrm{R}$. 
(iv) If $\mathrm{R}$ has at least one gr-n-ideal, then I is a graded weakly 2-absorbing primary ideal if and only if $\mathrm{I}$ is a graded 2-absorbing primary ideal of $\mathrm{R}$.

Proof. Straightforward.

Theorem 10 Let $\mathrm{R}$ be a $\mathrm{G}$-graded ring. Then $\mathrm{R}$ is a graded integral domain if and only if 0 is the only gr-n-ideal of $R$.

Proof. Let $\mathrm{R}$ be a graded integral domain. Assume that I is a nonzero gr-nideal of $R$. Then we have $I \subseteq \operatorname{Gr}(0)=0$ by Lemma 1 , a contradiction. Hence 0 is a gr-n-ideal by Example 1 (ii). Conversely, if 0 is the only gr- $n$-ideal, we get $\mathrm{Gr}(0)$ is a $\mathrm{gr}$-prime ideal and also a $\mathrm{gr}$-n-ideal by Corollary 1 and Theorem 8. Hence $\operatorname{Gr}(0)=0$ is a gr-prime ideal. Thus $R$ is a graded integral domain.

Theorem 11 Let $\mathrm{R}$ be a $\mathrm{G}$-graded ring and $\mathrm{J}$ be a graded ideal of $\mathrm{R}$ with $\mathrm{J} \cap(\mathrm{h}(\mathrm{R})-\mathrm{Gr}(0)) \neq \emptyset$. Then the following statements hold:

(i) If $\mathrm{I}_{1}$ and $\mathrm{I}_{2}$ are gr-n-ideals of $\mathrm{R}$ such that $\mathrm{I}_{1} \mathrm{~J}=\mathrm{I}_{2} \mathrm{~J}$, then $\mathrm{I}_{1}=\mathrm{I}_{2}$.

(ii) If IJ is a gr-n-ideal of $\mathrm{R}$, then $\mathrm{IJ}=\mathrm{I}$.

\section{Proof.}

(i) Suppose that $I_{1} J=I_{2} J$. Since $I_{2} J \subseteq I_{1}, J \cap(h(R)-G r(0)) \neq \emptyset$, and $I_{1}$ is a gr-n-ideal, by Theorem 2, we conclude that $I_{2} \subseteq I_{1}$. Similarly, since $I_{2}$ is a gr-n-ideal, we have the inverse inclusion.

(ii) It is clear from (i).

For G-graded rings $R$ and $R^{\prime}$, a G-graded ring homomorphism $f: R \rightarrow R^{\prime}$ is a ring homomorphism such that $f\left(R_{g}\right) \subseteq R_{g}^{\prime}$ for every $g \in G$.

The following result studies the behavior of $\mathrm{gr}$ - $n$-ideals under graded homomorphism.

Theorem 12 Let $\mathrm{R}_{1}$ and $\mathrm{R}_{2}$ be two $\mathrm{G}$-graded rings and $\mathrm{f}: \mathrm{R}_{1} \rightarrow \mathrm{R}_{2}$ a graded ring homomorphism. Then the following statements hold:

(i) If $\mathrm{f}$ is a graded epimorphism and $\mathrm{I}_{1}$ is a gr-n-ideal of $\mathrm{R}_{1}$ containing kerf, then $\mathrm{f}\left(\mathrm{I}_{1}\right)$ is a gr-n-ideal of $\mathrm{R}_{2}$.

(ii) If $\mathrm{f}$ is a graded monomorphism and $\mathrm{I}_{2}$ is a gr-n-ideal of $\mathrm{R}_{2}$, then $\mathrm{f}^{-1}\left(\mathrm{I}_{2}\right)$ is a gr-n-ideal of $\mathrm{R}_{1}$. 
Proof. (i) Suppose that $r_{g} s_{h} \in f\left(I_{1}\right)$ and $r_{g} \notin \operatorname{Gr}\left(0_{R_{2}}\right)$ for some $r_{g}, s_{h} \in$ $h\left(R_{2}\right)$. Since $f$ is onto, $f\left(x_{g}\right)=r_{g}, f\left(y_{h}\right)=s_{h}$ for some $x_{g}, y_{h} \in h\left(R_{1}\right)$. Hence $f\left(x_{g} y_{h}\right) \in f\left(I_{1}\right)$ implies that $x_{g} y_{h} \in I_{1}$ as Kerf $\subseteq I_{1}$. It is clear that $x_{g} \notin \operatorname{Gr}\left(0_{R_{1}}\right)$. Since $I_{1}$ is a gr-n-ideal of $R_{1}$, we conclude that $y_{h} \in I_{1}$; and so $s_{h}=f\left(y_{h}\right) \in f\left(I_{1}\right)$. Thus $f\left(I_{1}\right)$ is a gr-n-ideal of $R_{2}$.

(ii) Suppose that $r_{g} s_{h} \in f^{-1}\left(I_{2}\right)$ and $r_{g} \notin G r\left(0_{R_{1}}\right)$ for some $r_{g}, s_{h} \in h\left(R_{1}\right)$. Since kerf $=\{0\}$, we have $f\left(r_{g}\right) \notin G r\left(O_{R_{2}}\right)$. Since $f\left(r_{g} s_{h}\right)=f\left(r_{g}\right) f\left(s_{h}\right) \in I_{2}$ and $I_{2}$ is a gr-n-ideal of $R_{2}$, we conclude that $f\left(s_{h}\right) \in I_{2}$. It means $s_{h} \in f^{-1}\left(I_{2}\right)$, we are done.

Corollary 2 Let $\mathrm{I}_{1}$ and $\mathrm{I}_{2}$ be two graded ideals of a $\mathrm{G}$-graded ring $\mathrm{R}$ with $\mathrm{I}_{1} \subseteq \mathrm{I}_{2}$. Then the following statements hold:

(i) If $\mathrm{I}_{2}$ is a gr-n-ideal of $\mathrm{R}$, then $\mathrm{I}_{2} / \mathrm{I}_{1}$ is a gr-n-ideal of $\mathrm{R} / \mathrm{I}_{1}$.

(ii) If $\mathrm{I}_{2} / \mathrm{I}_{1}$ is a gr-n-ideal of $\mathrm{R} / \mathrm{I}_{1}$ and $\mathrm{I}_{1} \subseteq \mathrm{Gr}(0)$, then $\mathrm{I}_{2}$ is a gr-n-ideal of R.

(iii) If $\mathrm{I}_{2} / \mathrm{I}_{1}$ is a gr-n-ideal of $\mathrm{R} / \mathrm{I}_{1}$ and $\mathrm{I}_{1}$ is a gr-n-ideal of $\mathrm{R}$, then $\mathrm{I}_{2}$ is a gr-n-ideal of $\mathrm{R}$.

Proof. (i) Considering the natural graded epimorphism $\Pi: R \rightarrow R / I_{1}$, the result is clear by Theorem 12 .

(ii) Suppose that $r_{g} s_{h} \in I_{2}$ and $r_{g} \notin G r(0)$ for some $r_{g}, s_{h} \in h(R)$. Hence $\left(r_{g}+I_{1}\right)\left(s_{h}+I_{1}\right)=r_{g} s_{h}+I_{1} \in I_{2} / I_{1}$ and $r_{g} \notin \operatorname{Gr}\left(O_{R / I_{1}}\right)$. It implies that $s_{h}+I_{2} \in I_{1} / I_{2}$. Thus $s_{h} \in I_{1}$, we are done.

(iii) Let $I_{2} / I_{1}$ be a gr-n-ideal of $R / I_{1}$ and $I_{1}$ a gr-n-ideal of $R$. Assume that $I_{2}$ is not $g r-n$-ideal. Then $I_{1} \nsubseteq \operatorname{Gr}(0)$ by (ii). From Lemma 1 , we conclude that $I_{1}$ is not a $\mathrm{gr}$-n-ideal, a contradiction. Thus $\mathrm{I}_{2}$ is a $\mathrm{gr}$-n-ideal of $\mathrm{R}$.

Corollary 3 Let $\mathrm{R}$ be a $\mathrm{G}$-graded ring, I be a gr-n-ideal of $\mathrm{R}$ and $\mathrm{S}$ a subring of $\mathrm{R}$ with $\mathrm{S} \nsubseteq \mathrm{I}$. Then $\mathrm{I} \cap \mathrm{S}$ is a gr-n-ideal of $\mathrm{S}$.

Proof. Consider the injection $i: S \rightarrow R$. Then $i$ is a graded homomorphism. Since $I$ is a $g r-n$-ideal of $R, i^{-1}(I)=I \cap S$ is a gr-n-ideal of $S$ by Theorem 12 (ii).

Let $R$ be a G-graded ring and $S \subseteq h(R)$ a multiplicatively closed subset of $R$. Then graded ring of fractions is denoted by $S^{-1} R$ which defined by $S^{-1} R=$ $\oplus_{g \in G}\left(S^{-1} R\right)_{g}$ where $\left(S^{-1} R\right)_{g}=\left\{\frac{a}{s}: a \in R, s \in S, g=(\operatorname{deg} s)^{-1}(\operatorname{deg} a)\right\}$. A homogeneous element $r_{g} \in h(R)$ is said to be gr-regular if $\operatorname{ann}\left(r_{g}\right)=0$. 
Observe that the set of all gr-regular elements of $\mathrm{R}$ is a multiplicatively closed subset of R.

The following result studies the behaviour of $\mathrm{gr}$-n-ideal under localization.

Theorem 13 Let $\mathrm{R}$ be a G-graded ring, $\mathrm{S} \subseteq \mathrm{h}(\mathrm{R})$ a multiplicatively closed subset of $\mathrm{R}$. Then the following statements hold:

(i) If $\mathrm{I}$ is a $\mathrm{gr}-\mathrm{n}$-ideal of $\mathrm{R}$, then $\mathrm{S}^{-1} \mathrm{I}$ is a gr-n-ideal of $\mathrm{S}^{-1} \mathrm{R}$.

(ii) Let $\mathrm{S}$ be the set of all gr-regular elements of $\mathrm{R}$. If $\mathrm{J}$ is a gr-n-ideal of $\mathrm{S}^{-1} \mathrm{R}$, then $\mathrm{J}^{\mathrm{c}}$ is a gr-n-ideal of $\mathrm{R}$.

Proof. (i) Suppose that $\frac{a}{s} \frac{b}{t} \in S^{-1} I$ with $\frac{a}{s} \notin \operatorname{Gr}\left(0_{S^{-1} R}\right)$ for some $\frac{a}{s}, \frac{b}{t} \in$ $h\left(S^{-1} R\right)$. Hence there exists $u \in h(S)$ such that $u a b \in I$. Clearly, we have $a \notin \operatorname{Gr}(0)$. It implies that $u b \in I$; so $\frac{b}{t}=\frac{u b}{u t} \in S^{-1}$ I. Thus $S^{-1} I$ is a gr-n-ideal of $S^{-1} R$.

(ii) Suppose that $a, b \in h(R)$ with $a b \in J^{c}$ and $b \notin J^{c}$. Then $\frac{b}{1} \notin J$. Since $\mathrm{J}$ is a $\mathrm{gr}$ - $n$-ideal, we have $\frac{a}{1} \in \operatorname{Gr}\left(0_{S^{-1} R}\right)$. Hence $u a^{k}=0$ for some $u \in S$ and $k \geq 1$. Since $u$ is $g r$-regular, $a^{k}=0$; i.e. $a \in G r(0)$. Thus $J^{c}$ is a $g r-n$-ideal of $R$.

Definition 2 Let $\mathrm{S}$ be a nonempty subset of a $\mathrm{G}$-graded ring $\mathrm{R}$ with $\mathrm{h}(\mathrm{R})$ $\mathrm{Gr}(0) \subseteq \mathrm{S} \subseteq \mathrm{h}(\mathrm{R})$. Then we call $\mathrm{S}$ gr-n-multiplicatively closed subset of $\mathrm{R}$ if whenever $r_{g} \in h(R)-G r(0)$ and $s_{h} \in S$, then $r_{g} s_{h} \in S$.

Theorem 14 Let I be a graded ideal of a G-graded ring $\mathrm{R}$. Then the following statements are equivalent:

(i) I is a gr-n-ideal of $\mathrm{R}$.

(ii) $\mathrm{h}(\mathrm{R})-\mathrm{I}$ is a gr-n-multiplicatively closed subset of $\mathrm{R}$.

Proof. (i) $\Rightarrow$ (ii) Let I be a gr-n-ideal of R. Suppose that $r_{g} \in h(R)-\operatorname{Gr}(0)$ and $s_{h} \in h(R)-I$. Since $r_{g} \notin \operatorname{Gr}(0), s_{h} \notin I$, and I is a gr-n-ideal of R, we conclude that $r_{g} s_{h} \notin I$. Therefore $r_{g} s_{h} \in h(R)-I$. Since I is a gr-n-ideal of $R$, we have $\mathrm{I} \subseteq \operatorname{Gr}(0)$ by Lemma 1 . Then $h(R)-\operatorname{Gr}(0) \subseteq h(R)-I$.

(ii) $\Rightarrow$ (i) Suppose that $r_{g}, s_{h} \in h(R)$ with $r_{g} s_{h} \in I$ and $r_{g} \notin \operatorname{Gr}(0)$. If $s_{h} \in h(R)-I$, then from our assumption (ii), we have $r_{g} s_{h} \in h(R)-I$, a contradiction. Thus $s_{h} \in I$ which means that I is a gr-n-ideal of $R$.

Theorem 15 Let I be a graded ideal of a G-graded ring $\mathrm{R}$ and $\mathrm{S}$ a gr-nmultiplicatively closed subset of $\mathrm{R}$ with $\mathrm{I} \cap \mathrm{S}=\emptyset$. Then there exists a gr-n-ideal $\mathrm{K}$ of $\mathrm{R}$ such that $\mathrm{I} \subseteq \mathrm{K}$ and $\mathrm{K} \cap \mathrm{S}=\emptyset$. 
Proof. Let $\mathrm{D}=\{\mathrm{J}: \mathrm{J}$ is a graded ideal of $\mathrm{R}$ with $\mathrm{I} \subseteq \mathrm{J}$ and $\mathrm{J} \cap S=\emptyset\}$. Observe that $\mathrm{D} \neq \emptyset$ as $\mathrm{I} \in \mathrm{D}$. Suppose $\mathrm{J}_{1} \subseteq \mathrm{J}_{2} \subseteq \cdots$ is a chain in D. Then $\cup_{i=1}^{\infty} J_{i}$ is a gr$n$-ideal of $R$ by Lemma 2. Since $I \subseteq \cup_{i=1}^{\infty} J_{i}$ and $\left(\cup_{i=1}^{\infty} J_{i}\right) \cap S=\cup_{i=1}^{\infty}\left(J_{i} \cap S\right)=\emptyset$, we get $\cup_{i=1}^{\infty} J_{i}$ is the upper bound of this chain. From Zorn's Lemma, there is a maximal element $K$ of $D$. We show that this maximal element $K$ is a $g r-n-$ ideal of R. Suppose that $r_{g} s_{h} \in K$ and $s_{h} \notin K$ for some $r_{g}, s_{h} \in h(R)$. Then $K \subsetneq\left(K: R r_{g}\right)$. Since $K$ is maximal, it implies that $\left(K: R r_{g}\right) \cap S \neq \emptyset$. Hence there is an element $t_{\lambda} \in\left(K: R r_{g}\right) \cap S$. Then $r_{g} t_{\lambda} \in K$. If $r_{g} \in G r(0)$, then we are done. So assume that $r_{g} \notin \operatorname{Gr}(0)$. Since $S$ is gr-n-multiplicatively closed, we conclude that $r_{g} t_{\lambda} \in S$. Thus $r_{g} t_{\lambda} \in S \cap K$, a contradiction. Therefore $K$ is a gr-n-ideal of $R$.

\section{References}

[1] R. Abu-Dawwas, M. Bataineh, Graded r-ideals, Iran. J. Math. Sci. Inform (accepted).

[2] K. Al-Zoubi, R. Abu-Dawwas, S. Çeken, On graded 2-absorbing and graded weakly 2-absorbing ideals, Hacet. J. Math. Stat., 48 (3) (2019), $724-731$.

[3] K. Al-Zoubi, F. Qarqaz, An intersection condition for graded prime ideals, Boll Unione Mat. Ital., 11 (4) (2018), 483-488.

[4] K. Al-Zoubi, N. Sharafat, On graded 2-absorbing primary and graded weakly 2-absorbing primary ideals, J. Korean Math. Soc., 54 (2) (2017), 675-684.

[5] S. E. Atani, On graded weakly primary ideals, Quasigroups Related Systems, 13 (2) (2005), 185-191.

[6] C. Nastasescu, F. Van Oystaeyen, Graded and filtered rings and modules, Lecture notes in mathematics 758, Berlin-New York: Springer-Verlag, 1982.

[7] C. Nastasescu, F. Van Oystaeyen, Graded Ring Theory, Mathematical Library 28, North Holand, Amsterdam, 1982.

[8] C. Nastasescu and F. Van Oystaeyen, Methods of Graded Rings, LNM 1836. Berlin-Heidelberg: Springer-Verlag, 2004. 
[9] M. Refai, K. Al-Zoubi, On graded primary ideals, Turkish J. Math., 28 (3) (2004), 217-229.

[10] M. Refai, M. Hailat, S. Obiedat, Graded Radicals and Graded Prime Spectra, Far East Journal of Mathematical Sciences, part I (2000), 5973 .

[11] U. Tekir, S. Koc, K. H. Oral, n-ideals of Commutative Rings, Filomat, 31 (10) (2017), 2933-2941.

Received: October 18, 2018 